\title{
Coeducación: imprescindible en la formación de la Educación Infantil. El caso de la Psicología Evolutiva
}

\author{
Co-education: Essential in the development of early childhood education. \\ The case of evolutionary psychology
}
ISSN 2071-8748
E-ISSN 2218-3345
(c) $\$($
BY NC SA

URI: http://hdl.handle.net/11298/886

DOI: http://dx.doi.org/10.5377/entorno.v0i66.6731

\author{
Carmen Mañas-Viejo \\ Dpto. Psicología Evolutiva y Didáctica \\ Carmen.mavi@ua.es \\ ORCID: 0000-0002-4498-0974 \\ ORCID: 0000-0002-4498-0974 \\ Facultad de Educación \\ Universidad de Alicante España
}

Recibido: 14/08/18 Aprobado: 12/12/18

\section{Resumen}

La educación superior debe liderar el cambio cognitivo necesario en la estructura y organización del proceso de socialización de niños y niñas, con el doble objetivo de garantizar la igualdad de oportunidades que la escuela debe ofrecer $\mathrm{y}$ asimismo mejorar la calidad docente. La coeducación se nos presenta como un modelo imprescindible para lograrlo. Requiere, principalmente, la integración sistemática en el sistema educativo de la trasmisión del peso y valor de las mujeres en el mundo, $y$ de las diversas relaciones que entre los varones y las mujeres se pueden dar en él. Un elemento esencial en la coeducación es el lenguaje. Por ello hemos optado por introducir de manera sistemática y evaluable el uso de un lenguaje no sexista en las exposiciones orales y pruebas escritas de la asignatura de "Psicología Evolutiva de 0 a $3^{\prime \prime}$ años de edad que se imparte en el primer curso de Educación Infantil a lo largo del primer cuatrimestre. La metodología utilizada es integrar una estrategia

\section{Abstract}

Higher Education must lead to the necessary cognitive change in the structure and organization of the process of socialization of boys and girls, with the double objective of guaranteeing equal opportunities that the school must offer and, also, improve teaching quality. Co-education is presented as an essential model to accomplish it. This requires, mainly, methodic integration in the educational system of the transference of the importance and value of women in the world, and the diverse relationships that can exist between men and women. An essential element in co-education is language. Hence, we have chosen to adopt in a systematic and assessable way the use of nonsexist language in the speeches and written tests of the subject educative psychology from 0 to 3 years old that is taught in the first course of early childhood education through the first four-month period. The methodology implemented is to integrate a coeducational strategy, such as the use of non-sexist language, in research 
coeducativa, como el uso de un lenguaje no sexista, en un diseño de investigación en acción. Los resultados muestran un aumento del uso del lenguaje no sexista, así como una mayor sensibilización e interiorización, por parte de nuestro alumnado, de la importancia de la coeducación en general y del uso del lenguaje no sexista en particular.

\section{Palabras clave}

Estereotipo (psicología); Sociología de la educación; Psicología evolutiva; Educación de niños; Educación design in action. The results show an increase in the use of non-sexist language as well as greater awareness and internalization, by our student body, of the importance of co-education in general and the use of non-sexist language in particular.

\section{Keywords}

Stereotype (psychology), Sociology of Education, Evolutionary Psychology, Early Childhood Education, Education.

\section{Introducción}

Presentamos a continuación un caso práctico de introducción al lenguaje no sexista en la educación superior, en concreto, en el Grado de Educación Infantil, en la asignatura básica de Psicología Evolutiva de 0 a 3 años de edad, que se imparte en el primer curso durante el primer cuatrimestre. Consideramos imprescindible que la formación superior en educación (infantil, primaria y secundaria) y, más especialmente, las facultades de Educación lideren el cambio cognitivo necesario para avanzar hacia una sociedad más equitativa y solidaria con los sexos, los géneros, las razas y las clases sociales (Jiménez Rodrigo, Román y Traverso, 2011). Un modelo educativo imprescindible para avanzar en esa transformación necesaria es la coeducación, cuyo recorrido histórico en España estudia en profundidad Subirats desde la década de los 90, que resurge en España con fuerza (Subirats, 1994; 2006) hasta la actualidad (Subirats, 2010; Subirats y Tomé, 2010; Subirats, 2017) donde da cuenta rigurosa del viejo y persistente debate que para España es la educación en general y la coeducación en particular; y plantea los retos que esta tiene ante el futuro, entre los que señala la necesidad de integrar de manera rigurosa y sistemática en el debate educativo las evidencias teórico-empíricas. En esta línea, es interesante señalar los trabajos de Elena simón, que desde hace casi dos décadas trabaja en la elaboración y difusión de estrategias de aprendizaje basadas en el modelo coeducativo y sustentadas en la teoría feminista, invitando a reflexionar a la comunidad educativa y a la sociedad en general sobre el término igualdad (Simón, 2000; Simón, 2008; Simón, 2016; Venegas, 2017). La coeducación va más allá de compartir aula y currículum, pues permite educar en el respeto a la diversidad, apoyándose en los principios de equidad y cuidado. La coeducación aspira a crear nuevas identidades y representaciones (García Lastra, Calvo y Susinos, 2011) en una sociedad más inclusiva, más equitativa entre varones, mujeres y en sus posibles relaciones. Es allí, en el campo de las relaciones entre varones y mujeres, con los objetos del mundo, donde la coeducación puede producir los avances más notables (Mañas Viejo, Martínez Sanz, Molines, Montesinos, Sánchez, y Esquembre, 2016; Chamorro Giráldez, 2016); y itan necesarios!

La Psicología Evolutiva nos proporciona un amplio marco desde el que argumentar la coeducación: el construccionismo piagetiano - cuya teoría, no es baladí recordarlo, está basada en el estudio psicogenético de sus tres hijas- nos proporciona la contigüidad psicológica y biológica necesaria para conseguir dar lógica a la realidad física y mental en la que cada persona se desenvuelve. Sin embargo, este proceso no es suficiente; todo este proceso que requiere la construcción adaptativa ha de producirse dialécticamente (Vygotski, 1998); y es allí, en el discurso, en la comunicación dialéctica de ida y vuelta (Simón, Echeita, \& Sandoval, 2018), donde se construyen y reconstruyen los significados, donde la actividad comunicativa se convierte en instrumento estratégico para la maestra, para el maestro 
y para los niños y niñas que tratan de dar sentido y realidad al mundo que descubren a su alrededor. Todo lenguaje es interpretable; contribuye a nombrar, clasificar, pensar, construir representaciones y trasmitir la organización social del poder (Foucault, 1997; Butler, 2004; Jiménez Rodrigo et al., 2011). Aunque la comunicación y la cognición no sigan procesos psicológicos similares (Tirapu-Ustárroz, PérezSayes, Erekatxo-Bilbao y Pelegrín-Valero, 2007; Rivière, y Núñez, 2001), la alternativa no tiene que suponer una dicotomía. La actividad comunicativa se encuentra en la génesis de la representación y viceversa (Rodríguez y Moro, 1999). Así podemos afirmar que, mediante la dialéctica, conseguimos avances cognitivos, de igual modo que los avances cognitivos aumentan nuestro nivel dialéctico. Aun así, sabemos que sigue sin haber una comunicación buena y eficaz, en cualquier escenario, si no hay una simbiosis afectiva (Trevarthen, 1974, 1998 y 2018). Esto nos revela que el proceso de comunicación es algo más que palabras; es un proceso capaz de provocar avances cognitivos no solo para conocer nuestro mundo, sino también para comprender las relaciones que en él se dan. Por tanto, es muy importante el sentido, la interpretación, que de estas relaciones elípticas entre el aprendizaje y el desarrollo hagamos en las edades más tempranas, en las que el desarrollo deja huella neurológica. De ahí que subrayemos lo imprescindible que es el hecho de que nuestras maestras y maestros lleguen a la escuela sintiéndose capaces de moldear el aprendizaje, siempre de ida y vuelta elíptica, de quienes están bajo su responsabilidad. Es fundamental que quienes primero van a aportar significado a las experiencias de socialización de los niños y las niñas sean conscientes de la importancia de otorgar valor $\mathrm{y}$, por tanto, respeto y cuidado a cada persona; más, si cabe, durante los tres primeros años de edad, tan influyentes en la construcción del yo.

Para terminar de enmarcar teóricamente nuestro trabajo, no podemos dejar de reconocer y mencionar los estudios sobre mujeres, de género y feministas, que están consiguiendo ser tenidos en cuenta al señalar la importancia del lenguaje en la organización y construcción de las estructuras del pensamiento que guían la organización del mundo. Estos estudios han evidenciado cómo el uso y el contenido del lenguaje hípervisibiliza el género masculino e invisibiliza y desvaloriza el género femenino, legitimando la desigualdad de género (Cameron, 2005; Simón, 2010; Butler, y Preciado, 1997; Jiménez Rodrigo, Román y Traverso 2011). Esta reivindicación ha sido asumida por instituciones como universidades, ayuntamientos o el Parlamento Europeo
(2008), al reconocer que es ineludible la introducción de un lenguaje no sexista para lograr un avance real en el principio de igualdad de género. Por todo ello, nos parece de gran interés introducir, de forma sistemática, un uso consciente del lenguaje no sexista en la asignatura de Psicología Evolutiva de 0 a 3 años de edad. Así pues, centramos nuestro objetivo en conseguir que nuestro alumnado, mayoritariamente femenino, tome conciencia de la importancia del lenguaje en la comunicación y la trasmisión de significados en las edades más tempranas para que interiorice y use, en la medida de lo posible, el lenguaje no sexista como instrumento de cambio socio-cognitivo y de mejora de la calidad docente.

\section{Metodología}

Este trabajo sigue una metodología de investigación en acción (Giroux y Tremblay, 2004) y comienza en mayo de 2017, cuando el profesorado de la asignatura de Psicología Evolutiva de 0 a 3 años de edad realiza la última reunión de coordinación del curso (2016/17). En dicha reunión se revisan las incidencias, los problemas (si los ha habido), tanto respecto a la teoría como a los seminarios-taller y la evaluación. También se deciden y cierran las acciones de innovación teórico prácticas que se llevarán a cabo el curso siguiente. En esta reunión de mayo se decide llevar a cabo una acción innovadora, a la manera de experiencia piloto, con la introducción del uso oral y escrito de un lenguaje no sexista de manera sistemática -es decir, tanto en los objetivos como en los contenidos, procedimientos y en la evaluación - de la mencionada asignatura. Asumiendo, así, una estrategia dual y superando la controversia sobre el uso de una estrategia trasversal versus una específica. La guía docente recoge de forma trasversal la perspectiva de género. Al introducir el uso de un lenguaje no sexista de manera sistemática en una asignatura específica, asumimos plenamente el modelo coeducativo (DE COEDUCACIÓN, G.U.I.A, 2007).

Así pues, nos centramos en la asignatura de Psicología Evolutiva de 0 a 3 años de edad que se imparte en el primer curso del Grado de Infantil, durante el primer cuatrimestre, y en la Facultad de Educación de la Universidad de Alicante (España). La experiencia se llevará a cabo con dos de los siete grupos de matriculación existentes en la asignatura. Nuestro universo es la población estudiantil matriculada en la Facultad de Educación, y nuestra muestra está compuesta por 93 chicas y 7 chicos. En total son 100 chicas y chicos 
de entre 18 y 19 años, que entran en la universidad con la expectativa de ser maestras y maestros de niños y niñas, en este caso, de 0 a 3 años de edad.

\section{Diseño}

El diseño de este estudio tiene cuatro fases claras que se corresponden con las fases en las que desarrollamos la asignatura: presentación, justificación de los objetivos, desarrollo de los contenidos y evaluación.

Primera fase: presentación de la asignatura y de sus objetivos, entre los que se destaca, de forma explícita y justificada, la introducción del uso oral y escrito de un lenguaje no sexista, cuyas bases del marco teórico se exponen en el apartado anterior.

Segunda fase: breve interrogatorio dirigido y puesta en común. Por escrito responden individualmente a una serie de preguntas como: ¿Conoces el término de coeducación?; Define la coeducación con tus palabras; ¿Consideras importante la comunicación?; ¿Conoces el término “lenguaje no sexista"?; Define con tus palabras qué significa utilizar un lenguaje no sexista; ¿Crees que el género masculino incluye al género femenino?; ¿Crees que la utilización de un lenguaje determinado en la escuela tiene consecuencias? o ¿Consideras importante el uso de un lenguaje no sexista en el aula?, ¿ y en la familia y con tus amigos y amigas?

Tercera fase: desarrollo de los contenidos y reflexión sobre las principales manifestaciones del sexismo en el lenguaje (Sánchez Apellaniz, 2009:225), principalmente la hípervisibilidad del género gramatical masculino y el menosprecio cultural hacia el género gramatical femenino y sus consecuencias (Navarrete, 2018). Sin embargo, si somos conscientes de ello, podemos modificar nuestro lenguaje y transformar nuestra cultura, objetivo irrenunciable de la escuela y, por tanto, objetivo irrenunciable de la formación en el profesorado.

Cuarta fase: exposición y evaluación de un trabajo realizado en grupo, que deberá reflejar el uso del lenguaje no sexista, incluir el contenido concreto desarrollado en la asignatura —bien sobre la identidad, la comunicación, la representación, y la actividad física y mental—, y será expuesto en el aula. Además, cada estudiante deberá describir por escrito e individualmente su aportación concreta al trabajo.

\section{Evaluación del trabajo}

Nos proponemos evaluar la utilización de un lenguaje no sexista en las exposiciones orales y trabajos escritos que se han de realizar en la asignatura. Lo haremos prestando atención no solo a la forma (esfuerzo por visibilizar a varones y mujeres), sino también al contenido (esfuerzo por que el mensaje no sea sentencioso en relación con la identidad, el género, la clase y el rendimiento del receptor. Por ejemplo, siempre es mejor decir "me parece que has dicho una tontería" a decir "eres tonto". Tanto la morfosintaxis española como su riqueza de vocabulario toleran la integración de un uso no sexista del lenguaje sin dañar su gramática, y sin reproducir discriminación sexual, de género, raza y clase (Ayala, Guerrero y Medina, 2002).

Definimos los criterios de evaluación teniendo en consideración los objetivos propuestos, que no fuera generalizado el uso del masculino para referirse al conjunto de niños y niñas. Para definir este criterio, establecimos que más del $55 \%$ de las veces que se usa un genérico para referirse a un conjunto de chicas y chicos no fuera el masculino, tanto en la exposición como en la redacción del trabajo por evaluar (Jiménez Rodrigo, Román y Traverso 2011). La calificación obtenida por el uso de un lenguaje no sexista podría alcanzar el valor de un punto del total de cinco que se pueden obtener mediante la realización de las actividades prácticas sobre los contenidos teóricos explicados.

Por último, hay que comentar que las fases diseñadas se corresponden con la cronología planteada para la asignatura.

\section{Resultados y discusión}

Antes de comenzar la exposición y el análisis de los resultados obtenidos, queremos aclarar que, a pesar de haber recogido los datos disgregados por sexo, no esperamos ni buscamos en este trabajo obtener resultados significativamente distintos entre las mujeres y los varones, debido fundamentalmente a la excesiva desproporción entre los sexos y el evidente sesgo de género que tiene la educación en general y en concreto la educación infantil (Díaz y Morales, 2008; Rodríguez y Vila-Merino, 2016; Jiménez-Tejero, 2017).

Comenzaremos analizando los resultados obtenidos con el interrogatorio brevey dirigido y su puesta en voz alta. Mediante este breve e intencionado interrogatorio dirigido, deseamos 
obtener una fotografía, una revelación, del conocimiento intuitivo y experiencial que el alumnado manifiesta ante el tema que nos ocupa: la coeducación y el uso de un lenguaje no sexista. No obstante, para nuestra investigación en acción (Giroux y Tremblay, 2004), cada persona cuenta. Por último, conviene señalar que utilizamos este método de interrogatorio breve y dirigido como introducción para cualquier otro tema del contenido que desarrollamos en la asignatura.

Para analizar los resultados del interrogatorio dirigido, hemos diferenciado entre las respuestas dicotómicas, las conceptuales y las de opinión.

Tabla 1. Resultados sobre el interrogatorio dirigido: respuestas dicotómicas ofrecidos en frecuencias redondeadas

Sí \% NO \% NC \% Totales $\%$

\begin{tabular}{|l|l|l|l|l|}
\hline Preguntas & M V & M V & M V & Sí NO NC \\
\hline ¿Conoces el término coeducación? & 4399 & 500 & 70 & 46504 \\
\hline ¿Conoces el término lenguaje no sexista? & 89100 & 00 & 110 & 96014 \\
\hline $\begin{array}{l}\text { ¿Consideras importante la comunicación en el aula de 0 a 3 años } \\
\text { de edad? }\end{array}$ & 93100 & 00 & 70 & 9802 \\
\hline $\begin{array}{l}\text { ¿Crees que el uso de un lenguaje determinado tiene consecuencias } \\
\text { importantes en? }\end{array}$ & 92100 & 00 & 80 & 9307 \\
\hline ¿Crees que el género masculino debe seguir integrando al femenino? & 41100 & 250 & 340 & 442541 \\
\hline
\end{tabular}

Los resultados nos muestran que prácticamente podríamos partir al alumnado, entre quienes manifiestan conocer el término coeducación (46\%) y quienes manifiestan no conocerlo (50\%). Interpretaremos que el vaso está medio lleno, que vamos por buen camino, pero que debemos dedicar un tiempo a explicar bien el término y a justificar sus fundamentos en la intervención psicopedagógica, enfatizando en la importancia que las primeras experiencias tienen sobre nuestro desarrollo psicológico, biológico y social. (Lavados, 2012). El hecho de que casi el $54 \%$ del alumnado no conozca el término coeducación pone de manifiesto la necesidad de introducir estrategias coeducativas de manera directa, explícita, flexible, sistemática, planificada y evaluable en las asignaturas de la formación superior, en especial en la formación de educación infantil (Díaz, Morales, 2008; Jiménez-Tejero, 2017). No queremos dejar de señalar que prácticamente el $100 \%$ de los chicos (más del $99 \%$ manifiestan conocer el término coeducación, y que el $100 \%$ de quienes manifiestan no conocerlo, o no contestan, son chicas. El siguiente término por el que les preguntamos si lo conocen, o no, es lenguaje no sexista. El resultado muestra que el término es conocido por, prácticamente, la totalidad del alumnado (96\%), estando incluidos, de nuevo, el $100 \%$ de los chicos. Naturalmente, como explicamos al inicio de este apartado, no es relevante en este estudio profundizar en las posibles diferencias entre los sexos, pero sin duda es un dato interesante. En general, podemos atribuir el conocimiento del término por el alumnado al hecho de que tanto el modelo coeducativo como el uso, o no, de un lenguaje no sexista es un debate vivo en nuestra sociedad. En este sentido, afirma Subirats (2017:143): "No creo que la eficacia del cáncer sea sometida a debate en las tertulias de opinión de TV. Sin embargo, la educación se utiliza como arma arrojadiza entre partidos políticos con argumentaciones sin base empírica ni debate teórico". El estudio realizado por Barrios (2018), basado en la crítica feminista sobre el uso genérico del masculino y su visión androcéntrica del contenido en un medio de comunicación escrita, concluye que el uso generalizado que encuentra del masculino y la visión androcéntrica que proyecta su contenido perjudica tanto a los varones como a las mujeres, al reforzar la heteronormatividad. Por ello, podemos afirmar que el uso de un lenguaje no sexista no solo es beneficioso para las mujeres, también lo es para los varones.

Las siguientes preguntas: ¿Consideras importante la comunicación?, ¿Crees que el uso de un determinado lenguaje tiene consecuencias importantes en el aula de 0 a 3 años de edad? y, por último, ¿Crees que el género masculino debe de seguir integrando al género femenino?, pese a estar formuladas como preguntas de opinión, al incluir una opción de respuesta dicotómica, obliga al alumnado a posicionarse 
a favor de la pregunta o en contra. La opción no sabe/no contesta no consta en el interrogatorio, no se les da esa opción en el interrogatorio. Así encontramos que un 98 \% considera la comunicación en el aula un factor importante en la educación infantil. Encontramos, en la misma línea, una abrumadora mayoría ( $93 \%$ ) del alumnado que considera que el tipo de lenguaje utilizado en el aula de 0 a 3 años tiene consecuencias importantes. Sin embargo, prestaremos especial atención a ese $7 \%$, en su totalidad compuesto por chicas, que no tiene claro su importancia. La puesta en voz alta nos revela, sin entrar en debate, que buena parte de estas respuestas están sustentadas en la consideración de los bebés como personitas sin capacidad de análisis y cuyas necesidades primarias no incluyen la comprensión de lo que les rodea. Esperamos que el conocimiento de las teorías evolutivas (Piaget, Wallon, Vygotski...) y su relación con el aprendizaje les ayuden a modificar su concepto de desarrollo y aprendizaje, así como sus posibles relaciones.

Cuando analizamos las respuestas obtenidas en " $¿$ Crees que el género masculino debe incluir el femenino?", nos encontramos con un $41 \%$ que manifiesta que efectivamente el género masculino debe de seguir incluyendo el femenino; y casi otro $40 \%$ (en concreto el 38,7\%), que no contesta, no manifiesta opinión, todas ellas chicas; y casi un $25 \%$ que responde no, que no debe seguir incluyendo el género masculino al femenino. Los chicos, de nuevo al $100 \%$, están incluidos en la posición mayoritaria de no cambio. Esto evidencia, una vez más, cómo el pensamiento hegemónico imperante es el masculino, pues a pesar de ser franca minoría física, sus posiciones se integran en las creencias manifestadas por la mayoría, hegemónica, con la que coinciden
(Díaz y Morales, 2008; Rodríguez y Vila-Merino, 2016; JiménezTejero, 2017). Ello implica que ese casi $25 \%$ a favor del uso del lenguaje no sexista está integrado totalmente por chicas. La puesta en voz alta nos facilita atribuir las respuestas a favor del uso del genérico masculino, en la mayoría de los casos, a la economía y a la eficacia del lenguaje, pero también hay quienes lo atribuyen, aunque muy minoritariamente, a una moda, una tendencia que pasará.

Las dos siguientes cuestiones, "Define con tus palabras el término coeducación" y "haz lo mismo con el término lenguaje no sexista" demandan una respuesta abierta con la exigencia de definir con sus palabras los dos términos sobre los que estamos trabajando.

Resultados respuestas definición de conceptos: coeducación

Coeducación: hemos podido elaborar claramente dos grandes categorías excluyentes, pero no exclusivas, en las que englobar las respuestas obtenidas al definir el alumnado el concepto coeducación con sus palabras:

a) Definir la coeducación como la educación mixta, es decir, la educación que une en el aula y en el currículum a las niñas y a los niños, a los chicos y a las chicas. Esta categoría se puede enmarcar (de manera excluyente, pero no exclusiva) en el modelo de entender la coeducación equiparable a la escuela mixta (DE COEDUCACIÓN, G.U.I..A, 2007).

b) Definir la coeducación como la igualdad y universalidad del acceso a la educación para niños y niñas de manera global (Venegas, 2016).

Tabla 2. Resultados definición del concepto: coeducación

\begin{tabular}{l|l|l|l} 
Categorías & Mujeres & Varones & Total \\
\hline Educación mixta & 53 & 2 & 55 \\
\hline $\begin{array}{l}\text { Igualdad y } \\
\text { universalización del } \\
\text { acceso a la escuela }\end{array}$ & 31 & - & 31 \\
\hline Otras & 11 & & 11 \\
\hline Sin definición & 5 & - & 10
\end{tabular}


Los resultados nos muestran de nuevo la dicotomización que vamos fotografiando a lo largo de esta investigación en acción. Algo más de la mitad del alumnado, en donde se integra el $2 \%$ de los chicos que contestan, define la coeducación como una educación mixta para chicos y chicas, en línea con una de las concepciones más comunes de la coeducación. Es importante compartir saberes y espacios, pero tal y como dice Subirats (2017, p.145), "la escuela mixta es un éxito insuficiente". Más concretamente, Venegas (2016, p.15) dice: "La escuela mixta abre un espacio en el que escolariza conjuntamente a niños y niñas, sin embargo, no elimina los mecanismos de socialización genérica en el ámbito educativo". Un $31 \%$, entre quienes no hay ningún varón, define la coeducación señalando que su objetivo es la universalización del acceso a la escuela para todos los niños y las niñas del planeta. Un $11 \%$ la define como una moda, una tendencia más que se pasará; y, por último, vemos que un $10 \%$ no la define, y que dentro de quienes no la definen se integra la mayoría de los chicos y una minoría de las chicas. Pensamos que podemos estar frente a una barrera mental (Jiménez Rodrigo, Traverso Cortes, 2011). Ellos, por alguna razón, no hacen el esfuerzo de responder, sin duda enviando un mensaje negativo sobre la tarea en la que se está trabajando. La literatura revisada (Simón, 2008; Subirats 2010 y 2017; Chamorro Giráldez,
2016, entre otros estudios) nos señala la hipótesis del desinterés y desprestigio de la tarea. Nos preguntamos si el mensaje que subyace no es algo así como "vaya manera de perder el tiempo. Yo, paso". De cualquier forma, es muy reconfortante ver cómo el $86 \%$ del alumnado conoce los términos sobre los que vamos a trabajar y es capaz de expresar de forma escrita características que se pueden integrar en el modelo coeducativo, aunque no de manera completa. La coeducación necesita no solo un acceso universal a la escuela y un compartir saberes y espacios aún por conquistar, también debe reflejar con equidad las atribuciones de los saberes y sus consecuencias.

Resultados respuestas definición de conceptos: lenguaje no sexista

Las definiciones escritas por el alumnado nos han permitido agrupar sus respuestas en cinco grandes categorías excluyentes, pero no exclusivas:

Un lenguaje que nombra a las mujeres Un lenguaje atribuido a las feministas Un lenguaje inclusivo

Otras como Una moda

Sin definición

Tabla 3. Resultados respuestas definición de lenguaje no sexista

\begin{tabular}{l|l|l|l} 
Categorías & Mujeres \% & Varones \% & Total \% \\
\hline $\begin{array}{l}\text { Un lenguaje que } \\
\text { nombra a las mujeres }\end{array}$ & 51 & - & 51 \\
\hline Un lenguaje feminista & 10 & 2 & 12 \\
\hline $\begin{array}{l}\text { Un lenguaje inclusivo } \\
\text { de varones y mujeres }\end{array}$ & 31 & - & 31 \\
\hline Otras & 1 & & 1 \\
\hline Sin definición & - & - & 5
\end{tabular}

Vemos una correspondencia entre el $100 \%$ del alumnado que manifestó conocer el término lenguaje no sexista y el $94 \%$ que es capaz de definirlo de una forma que podemos considerar válida (que nombra a las mujeres, feminista e inclusivo). Sin embargo, solo un $25 \%$ del alumnado, también solo chicas, consideraba que el género masculino no tendría que incluir el género femenino. Este es un dato importante, ya que supone que aproximadamente un $50 \%$ de las chicas que conocen el término y lo saben definir, a la hora de ponerlo en práctica, de utilizar el lenguaje para visibilizar a las mujeres, sus saberes y relaciones, manifiestan en sus respuestas que el lenguaje sexista no es preciso que cambie. Esta argumentación tan poco lógica y conservadora es necesario explicitarla, visibilizarla, para comenzar a definir la barrera mental que les impide aplicar la lógica, que sin duda aplican en otras tareas, en el uso del lenguaje no sexista. Es todo un reto que descubran que el lenguaje también crea pensamiento, además de trasmitirlo 
(Butler, 2007; Lastra, 2010). Tendremos que trabajar en la toma de conciencia, en exigir una visión social crítica y feminista que, tal y como describe Elena simón en su libro Hijas de la igualdad, herederas de injusticias (2008), ayude a desvelar la carga sutil y pegajosa que trasmite el sexismo envuelto en cargas familiares, disponibilidad amorosa, responsabilidad en el cuidado de los otros... Para visibilizar esta realidad, nos apoyaremos en actividades de sensibilización e información sobre las consecuencias y la trascendencia que el uso de un lenguaje sexista tiene en el proceso de enseñanza aprendizaje, un proceso que, como hemos señalado anteriormente, es elíptico, de ida y vuelta.
Tan sólo un $1 \%$ lo define como un lenguaje de moda, pasajero; y un $5 \%$, en el que no hay chicas, no da ninguna definición. Observamos cómo solo un $20 \%$ de los chicos que manifestaron conocer los dos términos, coeducación y lenguaje no sexista, los definen. ¿Por qué? ¿Se trata de otra barrera mental o de la misma por la que las chicas manifiestan que las cosas deben seguir como están, aunque sea peor, no solo para ellas, sino también para ellos? (Barrios, 2018).

La última pregunta del interrogatorio dirigido tiene forma de encuesta y se responde con una escala tipo Likert con tan solo tres posibilidades de respuesta: mucha, poca, ninguna.

Tabla 4. Importancia del uso de un lenguaje no sexista

\begin{tabular}{l|l|l|l} 
Pregunta & $\begin{array}{l}\text { Muy } \\
\text { M\% V\% }\end{array}$ & $\begin{array}{l}\text { Poco } \\
\text { M\% V\% }\end{array}$ & $\begin{array}{l}\text { Nada } \\
\text { M\% V\% }\end{array}$ \\
\hline $\begin{array}{l}\text { ¿Crees que el uso de un lenguaje } \\
\text { no sexista es importante? }\end{array}$ & & & \\
\hline En el aula & 463 & 342 & 102 \\
\hline En la familia & 230 & 475 & 102 \\
\hline Con el grupo & 100 & 641 & 196
\end{tabular}

Con esta breve encuesta, les pedimos que se posicionen más específicamente sobre la importancia de utilizar un lenguaje no sexista en el aula de 0 a 3 años de edad. Nuevamente, observamos que se percibe esa fotografía en la que cerca de un $50 \%$ tiene claro la importancia de seguir un modelo coeducativo y del uso estratégico de un lenguaje no sexista, pero solo un $25 \%$ parece que lo tiene interiorizado y manifiesta una mejor aptitud y actitud para utilizarlo en su vida no solo académica, sino también cotidiana, pues es allí donde debe producirse la verdadera transformación (Trevarthen, 2018).

Resultados obtenidos en la evaluación

Antes de exponer los resultados de la evaluación, queremos recordar que la evaluación se ha realizado sobre las exposiciones orales grupales y los trabajos escritos que han tenido que realizar durante la asignatura. Los resultados obtenidos fueron realmente satisfactorios.

Tabla 5. Resultados evaluación del uso de un lenguaje no sexista en las exposiciones orales y escritas sometidas a evaluación

\begin{tabular}{l|l|l} 
Uso & $\mathrm{M} \% \mathrm{~V} \%$ & Total $\%$ \\
\hline Oral & 88100 & 94 \\
\hline Escrito & 352 & 37 \\
\hline Ninguno & $2-$ & 6
\end{tabular}


El $94 \%$ del alumnado, incluido el $100 \%$ de los chicos, utiliza en los procesos evaluativos de manera oral y, o, escrita el lenguaje no sexista, aunque solo el $37 \%$ lo hace tanto en las exposiciones orales como en los trabajos escritos sujetos a evaluación. Es interesante resaltar que quienes lo utilizan por escrito lo usan también en las exposiciones orales, mientras que no sucede lo contrario. Esta situación la podemos atribuir, entre otras causas, al refuerzo que tiene el alumnado al realizar la exposición oral de su trabajo, mientras se le recuerda al inicio de esta y también cuando alguna persona del grupo utiliza el genérico masculino, que valoramos que el $55 \%$ de las veces se use un genérico no masculino, con el objetivo de visibilizar a todas las personas que comparten el aula, ellos y ellas.

El $57 \%$ del alumnado utiliza el lenguaje no sexista en sus exposiciones orales, pero no en los trabajos entregados para su evaluación por escrito. Estos datos nos hacen pensar que, a pesar del uso oral, la interiorización del lenguaje no sexista está aún lejos; y es preciso insistir tanto en la sensibilización como en actuaciones concretas (Vila-Merino, 2016). Por otra parte, no debemos obviar que el $5 \%$, mayoritariamente masculino, no ha utilizado el lenguaje no sexista en ninguna de las actividades evaluables. En esta ocasión, de nuevo, la mayoría de los chicos responde de manera radicalmente conservadora. Sin embargo, no es la respuesta mayoritaria; las chicas cambian la educación (Lastra, 2010; Lastra, Salvador y Rada, 2008). Por último, solo resta manifestar que nos satisface comprobar cómo ese $25 \%$ que ya estaba abierto a un uso del lenguaje no sexista ha aumentado casi en un $15 \%$ en un solo cuatrimestre.

\section{Conclusiones}

Conscientes de las limitaciones que tiene una investigación en acción y de su pequeña proyección, no podemos dejar de subrayar que los resultados obtenidos avalan la idea de liderar desde la universidad, y en concreto desde las facultades de Educación, el cambio cognitivo imprescindible que requiere la escuela para dejar de ser, de manera generalizada, un mero trasmisor y comenzar a creer en sus propias posibilidades de trasformación social. La primera conclusión que se ha de señalar es que los resultados muestran que la utilización de una estrategia dual (trasversal y específica) en la introducción de un lenguaje no sexista ha resultado satisfactoria, dentro de una metodología de investigación en acción, en tanto en cuanto hemos logrado aumentar su uso en un $15 \%$ en el alumnado en general. Ello confirma la eficacia de un modelo coeducativo en el logro de la transformación cultural del aula. Por otra parte, la elección de la asignatura de Psicología Evolutiva y sus contenidos teórico-prácticos, así como el diseño participativo, dialéctico y constructivo llevado a cabo, han posibilitado la profundización necesaria sobre la importancia de una comunicación temprana de socialización no sexista en el aula. Nos parece muy relevante resaltar el conocimiento mayoritariamente acertado sobre el uso de un lenguaje no sexista de nuestro alumnado, lo que sin duda ha facilitado mucho la investigación-acción realizada.

Los resultados obtenidos con el brevey dirigido interrogatorio sobre la coeducación y el uso de un lenguaje no sexista nos han permitido tener en las primeras sesiones de clase una fotografía del nivel de conocimiento intuitivo y experiencial del conjunto del alumnado sobre la coeducación, así como sobre el uso y la importancia que otorgan a la utilización de un lenguaje no sexista en el aula, en la familia y en el grupo de amigos y amigas. Esa fotografía nos ha guiado en la ejecución del desarrollo de nuestro programa de introducción teórico-práctico de un lenguaje no sexista, ajustando los temas y las actividades que se debían desarrollar sobre el uso de un lenguaje no sexista en los seis bloques que componen la asignatura. Así decidimos que explicaríamos con detenimiento la adquisición del lenguaje en el primer bloque, especialmente a la hora de explicar el entorno sociocultural como uno de los factores que tener en cuenta en el desarrollo de ellos y de ellas. Avanzaremos en la adquisición del lenguaje en el cuarto bloque, en el que tratamos las claves del desarrollo cognitivo, especialmente en el tema del conocimiento de la realidad y exploración del mundo físico, natural y social. Continuaremos con la explicación de las funciones del lenguaje en el quinto bloque, en el que abordamos las claves del desarrollo afectivo y emocional, en concreto en el tema en el que nos detengamos en la construcción de la identidad y el papel del género en el descubrimiento del yo. Finalmente, en el sexto bloque, donde abordamos los inicios de la comunicación y el lenguaje, trataremos de reforzar la importancia del uso de un lenguaje no sexista en el proceso de socialización y sus productos y pondremos de relevancia el juego, la pedagogía lúdica, como guía de cualquier actividad, también en la comprensión del uso de un lenguaje no sexista.

Los resultados obtenidos nos alientan a proponer la introducción del lenguaje no sexista en los siete grupos matriculados de la asignatura, aunque antes de ello debemos 
asegurarnos de que el profesorado que se va a hacer cargo de la asignatura tiene las aptitudes y actitudes necesarias para llevarlo a cabo. Nos ocuparemos también de mejorar el diseño de la investigación en acción y trataremos de realizar un seguimiento más riguroso de los avances logrados. Sin lugar a dudas, intentaremos ampliar el número de grupos a quienes podemos formar con rigor en un modelo coeducativo utilizando la introducción al lenguaje no sexista como herramienta de potente cambio sociocognitivo.

\section{Referencias}

Berríos, P. (2018). “El sistema de prestigio en las universidades y el papel que ocupan las mujeres en el mundo académico". Calidad en la Educación, (23).

Butler, J., \& Preciado, B. (1997). Lenguaje, poder e identidad (no $306.44 \mathrm{~B} 8$ ).

Butler, J. (2007). El género en disputa: el feminismo y la subversión de la identidad. Paidós.

Chamorro Giráldez, L. (2016). "El sexismo en el lenguaje. La política contemporánea".

DE COEDUCACIÓN, G.U.I..A. (2007). "Documento de síntesis sobre la Educación para la Igualdad de oportunidades entre Mujeres y Hombres" [en línea]. Red2 Red Consultores SL Instituto de la Mujer. Observatorio para la Igualdad de Oportunidades, 15-20.

Díaz, A.R., \& Morales, P.A. (2008). "Género y la formación del profesorado en los estudios de Educación Infantil". Revista electrónica interuniversitaria de formación del profesorado, 11(3), 5.

Foucault, M. (1997). La arqueología del saber. Siglo xxi.

Giroux, S., \& Tremblay, G. (2004). Metodología de las ciencias humanas: la investigación en acción. Fondo de Cultura Económica.

Jiménez Rodrigo, M.L.; Román Onsalo, M., \& Traverso Cortes, J. (2011). Lenguaje no sexista y barreras a su utilización. Un estudio en el ámbito universitario. Revista de investigación en educación, 2(9), 174-183.

Jiménez-Tejero, L. (2017). "Coeducar en Educación Infantil". En https://hdl.handle.net/10953.1/5201

Lastra, M.G.; Salvador, A.C., \& Rada, T.S. (Eds.). (2008). Las mujeres cambian la educación: investigar la escuela, relatar la experiencia (Vol. 49). Narcea Ediciones.

Lastra, M.G. (2010). "La voz de las mujeres en la universidad". Revista de la Asociación de Sociología de la Educación (RASE), 3(3), 357-368.
Lavados, J. (2012). “La neurobiología del aprendizaje temprano". Estudios Sociales, 120.

Llorenç, E., \& Rodríguez, M.E.S. (2016). María Elena Simón: "L'escola coeducativa no progressa adequadament". Futura, (32), 15-20.

Lemus, J. (2006). "¿El mito del español amenazado?". Científica, 7, 54-73.

Mañas Viejo, C.; Sanz, A.M.; Alcaraz, M.M.; Sánchez, M.N.M., \& Esquembre, M.M. (2016). "Coeducación y cambio conceptual". En XIV Jornadas de Redes de Investigación en Docencia Universitaria. Investigación, innovación y enseñanza universitaria. Enfoques pluridisciplinares (pp. 2381-2391). Instituto de Ciencias de la Educación.

Navarrete Fonseca, C.S. (2018). “Expresiones socioculturales en la identidad de género de los niños y niñas de 3 a 5 años de la Unidad Educativa José Joaquín Olmedo" (Bachelorss thesis, Universidad Técnica de Ambato. Facultad de Ciencias Humanas y de la Educación. Maestría en Educación Inicial).

Rodríguez, M.D.C., \& Vila-Merino, E.S. (2016). "Educación y género en la formación docente en un enfoque de equidad y democracia".

Román Onsalo, M.; Jiménez Rodrigo, M. L.; Traverso Cortés, J.; Gil Galván, M.R.; Gil Galván, F.J., \& Sánchez-Apellaniz García, M. (2009). Manual de agentes de igualdad.

Sánchez-Apellaniz García (2009). Manual de Igualdad. Diputación de Sevilla

Simón, E. (2000). “Tiempos y espacios para la coeducación". El harén pedagógico, 33-51.

Simón, M.E. (2008). Hijas de la igualdad, herederas de injusticias. Madrid: Narcea.

Simón, C.; Echeita, G., \& Sandoval, M. (2018). "Incorporating students' voices in the 'Lesson Study'as a teachertraining and improvement strategy for inclusión" / "La incorporación de la voz del alumnado a la 'Lesson Study'como estrategia de formación docente y mejora para la inclusión". Cultura y Educación, 30(1), 205-225.

Subirats, M. (2006). "La educación de las mujeres: de la marginalidad a la coeducación. Propuestas para una metodología de cambio educativo". C. Rodríguez (Comp.), Género y currículo. Aportaciones del género al estudio y práctica del currículum, 229-255. Subirats, M. (2010). ¿Coeducación o escuela segregada? Un viejo y persistente debate. Revista de la Asociación de Sociología de la Educación (RASE), 3(1), 143-158. 
Subirats, M., \& Tomé, A. (2010). Balones fuera: reconstruir los espacios desde la coeducación. Octaedro.

Subirats, M. (2017). Coeducación, apuesta por la libertad. Octaedro.

Susinos, T.; Calvo, A.; Rojas, S.; Lázaro, S.; García Lastra, M.; Haya, I., \& Rodríguez-Hoyos, C. (2010). "La voz del alumnado como estrategia de mejora de la escuela". En Manzanares, MA [coord.] (2010): Organizar y dirigir en la complejidad: instituciones educativas en evolución, pp. 282-291. Madrid. Wolters Kluwer

Tirapu-Ustárroz, J.; Pérez-Sayes, G.; Erekatxo-Bilbao, M., \& Pelegrín-Valero, C. (2007). "¿Qué es la teoría de la mente?". Revista de neurología, 44(8), 479-489.

Rivière, Á., \& Núñez, M. (2001). La mirada mental: desarrollo de las capacidades cognitivas interpersonales. Aique.

Rodríguez, C., \& Moro, C. (1999). El magico numéro très. Cuando les ninos aûn no hablan. Barcelona. Paidós.

Rodríguez, M.D.C., \& Vila-Merino, E.S. (2016). "Educación y género en la formación docente en un enfoque de equidad y democracia".

Trevarthen, C. (1979). "Communication and cooperation in early infancy:A description of primary intersubjectivity". Before speech: The beginning of interpersonal communication, 1, 530-571.
Trevarthen, C. (1998). "The concept and foundations of infant intersubjectivity". Intersubjective communication and emotion in early ontogeny, 15-46.

Trevarthen, C. (2018). "Sharing joyful friendship and imagination for meaning with infants, and their application in early intervention". In Surviving the Early Years, 19-55. Routledge.

Trevarthen, C. (2011). "La Psicobiología Intersubjetiva del Significado Humano: El aprendizaje de la cultura depende del interés en el trabajo práctico cooperativo y del cariño por el gozoso arte de la buena compañía". Clínica e Investigación Relacional, 5 (1), 17-33.

Velasco Marugán, N. (2012). "Estudio sobre coeducación en el aula de educación infantil".

Venegas, M. (2017). "Coeducar las relaciones afectivo sexuales para promover la igualdad sexual y de género y la justicia social". Revista Internacional de Educación para la Justicia Social, 6(2).

Venegas, M. y Heras, P. (2016). “Financiar la segregación educativa: Un debate sobre la LOMCE desde una perspectiva crítica de género". Revista Educación, Política y Sociedad, 1(2), 73-99.

Vigotsky, L.S. (1988). "Interacción entre enseñanza y desarrollo". Selección de Lecturas de Psicología de las Edades I, 3. 